Artículo

\title{
Frecuencia de Cryptosporidium en perros asociados a establos lecheros y en áreas urbanas del estado de Aguascalientes, México
}

\author{
Irene Vitela-Mendoza ${ }^{\mathrm{a}^{*}}$ \\ Kenia Padilla Díaz ${ }^{\mathrm{a}}$ \\ Carlos Cruz-Vázquez \\ Leticia Medina-Esparza ${ }^{\mathrm{a}}$ \\ Miguel Ramos-Parra ${ }^{\mathrm{a}}$
}

${ }^{a}$ Instituto Tecnológico El Llano Aguascalientes, Km 18 Carretera Ags-SLP., Municipio de El Llano, Ags., 20330, Aguascalientes, México.

* Autor de correspondencia: vitelairene@yahoo.com.mx

\section{Resumen:}

El objetivo del estudio fue determinar la frecuencia de Cryptosporidium spp, así como llevar a cabo la identificación de algunos factores de riesgo asociados a la infección en perros asociados a establos lecheros en Aguascalientes, México, y en perros procedentes del área urbana de la capital del mismo estado. Se colectaron muestras de excremento de 168 perros domiciliados en 30 establos lecheros distribuidos en diez municipios del estado, y de 144 perros residentes del Centro de Control, Atención y Bienestar Animal (CCABA), del municipio de Aguascalientes (área urbana), las cuales se procesaron mediante frotis fecal teñido con Kinyoun para identificar la presencia de ooquistes del parásito. Se levantó una encuesta para identificar diferentes características de los individuos y se realizó un análisis de riesgos mediante regresión logística. La frecuencia general de perros infectados por Cryptosporidium spp., fue $20.5 \%$ (64/312; IC95\% 1625), mientras que en los perros procedentes del CCABA fue $9 \%$ (13/144; IC95\% 5-15), y en los asociados a establos fue de $30 \%$ (51/168; IC95\% 23-38). El $70 \%$ de los establos tuvieron animales positivos, mientras que el promedio de perros por establo fue de 5.6, y la densidad fue de 2 a 12 perros. Se identificó como factor de riesgo a la infección por 
Cryptosporidium a la variable excremento diarreico, tanto en los perros de origen urbano (OR, 3.2; IC95\% 1.06-9.79 $P<0.03$ ) como en los asociados a los establos (OR, 2.7; IC95\% 1.36-5.49 $P<0.001)$. En ninguna de las otras variables analizadas fue posible identificar una asociación estadísticamente significativa.

Palabras clave: Cryptosporidium, Frecuencia, Perros, Factores de riesgo.

Recibido: 29/01/2018

Aceptado: 06/03/2018

\section{Introducción}

La criptosporidiosis es una parasitosis intestinal causada por protozooarios del género Cryptosporidium (Apicomplexa: Cryptosporiidae); a diferencia de otros coccidios los ooquistes de Cryptosporidium spp, son infectantes desde el momento de ser excretados por los individuos afectados y pueden infectar a otro huésped al tiempo de ser ingeridos. Este protozoario es de distribución cosmopolita, considerado como parásito oportunista, causante de trastornos digestivos severos en individuos jóvenes, afecta a numerosas especies de animales domésticos, silvestres y al ser humano ${ }^{(1)}$. La tendencia actual a poseer mascotas, particularmente perros y gatos es común en todo el mundo; el contacto con los animales produce un vínculo afectivo y ayuda a los niños en su desarrollo emocional, aunque los perros están expuestos a numerosas infecciones parasitarias, representando un riesgo de transmisión tanto a otros animales como a los humanos ${ }^{(2)}$. Los perros pueden ser naturalmente infectados por C. canis $^{(3)}$, C. parvum $^{(4)}$, C. $_{\text {meleagridis }}{ }^{(5)}$ y $C$. muris $^{(6)}$; generalmente la infección es asintomática; sin embargo en ocasiones puede cursar con manifestaciones clínicas severas que incluyen diarrea acuosa, fiebre y puede afectar el aparato respiratorio, hígado y páncreas, especialmente en animales inmunocomprometidos ${ }^{(7,8)}$.

La criptosporidiosis canina tiene una amplia distribución y se ha reportado en perros con dueño, en criaderos, en refugios, así como en perros callejeros, principalmente en áreas urbanas y en algunas comunidades rurales de diversas partes del mundo ${ }^{(9,10,11)}$. En México, existe escasa información sobre los rasgos epidemiológicos de esta parasitosis en poblaciones de perros asociadas a las áreas y comunidades urbanas y rurales, que permita comprender su potencial participación en la transmisión y mantenimiento de la 
infección tanto a otros animales domésticos, como al ganado bovino, así como al ser humano.

El objetivo de este estudio fue determinar la frecuencia de Cryptosporidium spp, así como llevar a cabo la identificación de algunos factores de riesgo asociados a la infección, en una muestra de perros de ambiente rural asociados a establos lecheros en Aguascalientes, México, y otra de perros procedentes del área urbana de la capital del mismo estado.

\section{Material y métodos}

El estudio se realizó en el estado de Aguascalientes, que se encuentra localizado a $21^{\circ} 37^{\prime}$ y $22^{\circ} 01^{\prime} \mathrm{N}$ y $101^{\circ} 52^{\prime}$ y $102^{\circ} 35^{\prime} \mathrm{O}$, con altitud entre 1,765 y $2,400 \mathrm{msnm}$, temperatura promedio anual de $17.4{ }^{\circ} \mathrm{C}$, y precipitación pluvial media anual de $526 \mathrm{~mm}$, que se concentra en el verano ${ }^{(12)}$.

\section{Sitios de muestreo de perros asociados a establos lecheros}

Se seleccionaron por conveniencia, 30 establos lecheros distribuidos en 10 municipios del estado de Aguascalientes. En cada uno de los municipios se seleccionaron tres establos considerando que contara al menos con un perro domiciliado, y que el propietario proporcionara las facilidades pertinentes para desarrollar el trabajo. 
Figura 1: Localización de los establos incluidos en el estudio, los cuales están señalados con los números 1, 2 y 3 en cada municipio, así como del CCABA

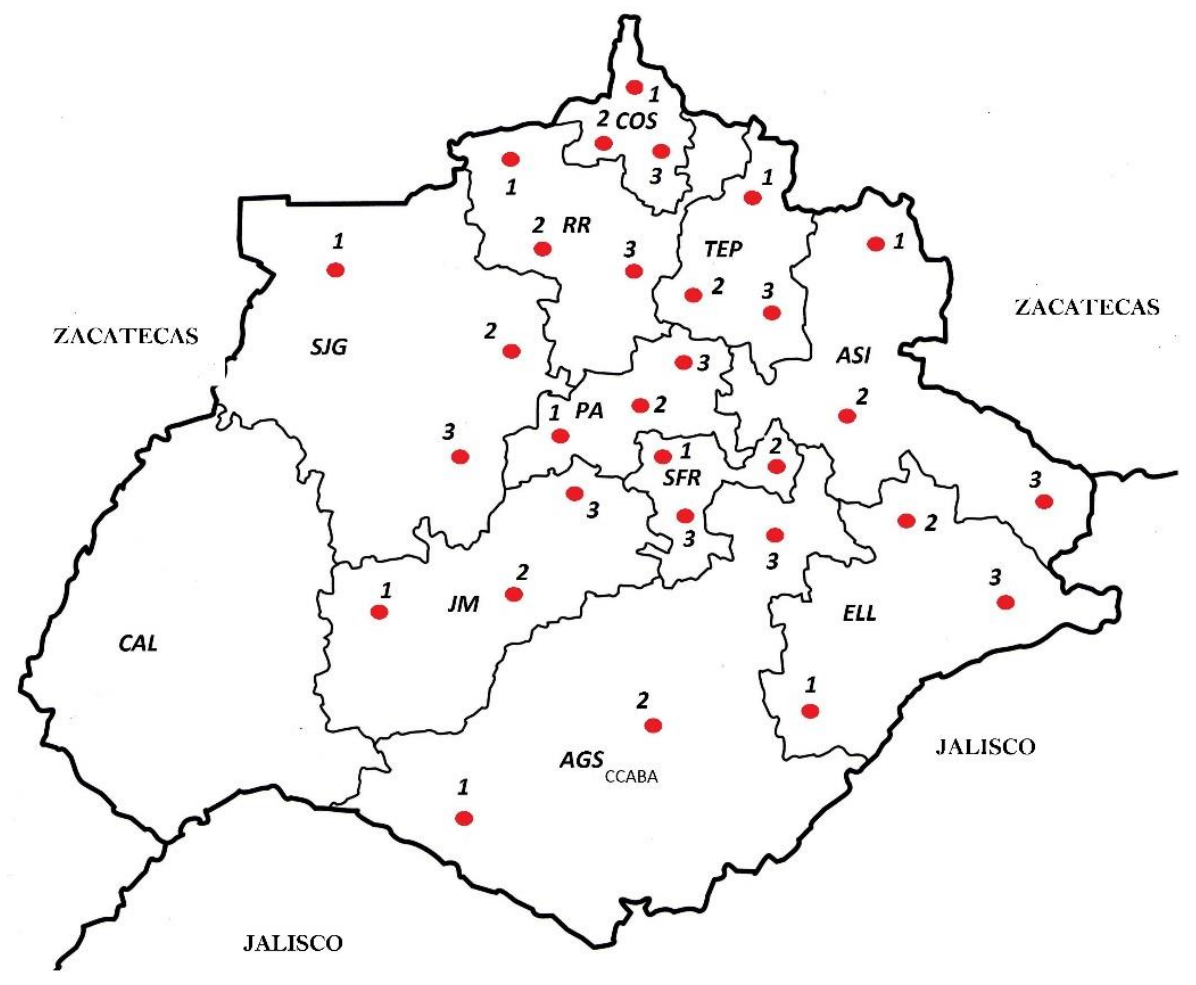

Los municipios son: Pabellón de Arteaga (PA), Asientos (ASI), San José de Gracia (SJG), Cosío (COS), Tepezalá (TEP), Rincón de Romos (RR), San Francisco de los Romo (SFR), Calvillo (CAL), Jesús María (JM), Aguascalientes (AGS) y El Llano (ELL).

\section{Sitio de muestreo de perros del área urbana}

Esta actividad se desarrolló en las instalaciones del Centro de Control, Atención y Bienestar Animal del municipio de Aguascalientes (CCABA), sitio en que se alojan perros sin dueño que son recogidos de las calles del área urbana, así como aquellos que son abandonados por sus propietarios en estas instalaciones. De acuerdo a las normas y procedimientos de CCABA, los perros que no son reclamados en el término de $72 \mathrm{~h}$, son sacrificados humanitariamente de acuerdo con la Norma Oficial Mexicana NOM-033SAG/ZOO-2014. 


\section{Toma de muestras}

Los establos lecheros se visitaron por una sola ocasión, colectando una muestra de excremento de aproximadamente $25 \mathrm{~g}$, de cada uno de los perros, mismos que se mostraban clínicamente sanos; en total se obtuvieron 168 muestras. El CCABA se visitó una vez por semana durante un periodo de tres meses; en cada ocasión se eligieron al azar 12 perros clínicamente sanos de los cuales se tomó una muestra de excremento previo a su sacrificio; en total se obtuvieron 144 muestras. Las muestras se trasladaron al laboratorio en condiciones de refrigeración para ser procesadas el mismo día de la colecta, y se acompañaron de una encuesta con datos de identificación del perro, tales como el sexo, edad (mediante la revisión de la dentición), así como de la consistencia de la muestra de excremento (firme/diarreica); en los animales asociados a establos, se registró además información sobre la alimentación (seco balanceado/preparado en casa/combinado), sitio donde beben agua (bebederos exclusivos de los perros/compartidos con otras especies), y el programa de medicina preventiva (vacunación/desparasitación).

\section{Diagnóstico parasitoscópico}

Las muestras se procesaron de acuerdo a lo descrito por Castillo et al ${ }^{(13)}$, tomando, en cada caso, $10 \mathrm{~g}$ de excremento diluyendo muestra 1:1 con agua oxigenada, preparando seis frotis fecales sobre una laminilla. Después se dejó secar por $24 \mathrm{~h}$ a temperatura ambiente y se tiñeron mediante la técnica de tinción ácido-alcohol resistente de Kinyoun, y se observó el frotis en un microscopio (LCD Digital, Leica®) a 100X. Con la finalidad de minimizar las lecturas de falsos positivos, la muestra se consideró positiva cuando se observaron al menos $\geq 5$ ooquistes de Cryptosporidium spp., después de revisar los seis frotis (los ooquistes se presentan de forma esférica y teñidos de color rojo a rosa pálido). 


\section{Análisis de la información}

Se calculó la frecuencia de perros positivos a Cryptosporidium spp., en base al resultado del examen parasitoscópico en la muestra total y de acuerdo a diferentes características de los perros en las dos poblaciones estudiadas. Se realizó un análisis de riesgos mediante regresión logística ${ }^{(14)}$, en donde la variable dependiente fue el estado de infección parasitario y las variables independientes se seleccionaron por el método "backward step by step", y según la prueba de Ji cuadrada, se excluyeron las variables no significativas $(P<0.05)$. Las posibilidades de riesgo $(\mathrm{OR})$ se estimaron para las variables independientes que mostraron significancia estadística en el análisis multivariado $(P<0.05)$. El análisis se desarrolló con el programa Statistics Data Analysis (STATA) v. 9.1

\section{Resultados}

La frecuencia general de perros infectados con Cryptosporidium spp., diagnosticada mediante el análisis parasitoscópico, fue de $20.5 \%$ (64/312; IC95\% 16-25), mientras que en los perros procedentes del área urbana del municipio de Aguascalientes fue de $9 \%$ (13/144; IC95\% 5-15), y en los asociados a establos lecheros de $30 \%$ (51/168; IC95\% 23-38).

La frecuencia de la infección por Cryptosporidium spp., de acuerdo al municipio de origen de los perros asociados a establos lecheros se muestra en el Cuadro 1. En el mismo se puede observar que la frecuencia más alta se registró en el municipio de Jesús María (58\%), mientras que la menor lo fue en el de Cosío (15\%). El $100 \%$ de los municipios estudiados tuvieron animales positivos, mientras que solo $70 \%$ de los establos (21/30), tuvieron animales positivos. El promedio de perros por establo fue de 5.6, y la densidad fue de 2 a 12 perros. 
Cuadro 1: Distribución de la infección por Cryptosporidium spp., en diez municipios del estado de Aguascalientes, México

\begin{tabular}{|c|c|c|c|c|}
\hline Municipio/Establo & $\mathbf{n}$ & Positivas & Frecuencia (\%) & IC 95\%* \\
\hline Aguascalientes & 18 & 4 & \multirow{4}{*}{22} & \multirow{4}{*}{$7-48$} \\
\hline 1 & 9 & 4 & & \\
\hline 2 & 5 & - & & \\
\hline 3 & 4 & - & & \\
\hline Asientos & 14 & 3 & \multirow{4}{*}{21} & \multirow{4}{*}{$5-51$} \\
\hline 1 & 7 & 2 & & \\
\hline 2 & 2 & - & & \\
\hline 3 & 5 & 1 & & \\
\hline Cosío & 13 & 2 & \multirow{4}{*}{15} & \multirow{4}{*}{$2-46$} \\
\hline 1 & 2 & 1 & & \\
\hline 2 & 6 & 1 & & \\
\hline 3 & 5 & - & & \\
\hline El Llano & 31 & 12 & \multirow{4}{*}{39} & \multirow{4}{*}{$22-57$} \\
\hline 1 & 12 & 6 & & \\
\hline 2 & 10 & 4 & & \\
\hline 3 & 9 & 2 & & \\
\hline Jesús María & 19 & 11 & \multirow{4}{*}{58} & \multirow{4}{*}{$33-78$} \\
\hline 1 & 2 & 2 & & \\
\hline 2 & 8 & 6 & & \\
\hline 3 & 9 & 3 & & \\
\hline Pabellón de Arteaga & 22 & 4 & \multirow{4}{*}{18} & \multirow{4}{*}{$6-41$} \\
\hline 1 & 11 & 2 & & \\
\hline 2 & 6 & 2 & & \\
\hline 3 & 5 & 0 & & \\
\hline Rincón de Romos & 11 & 4 & \multirow{4}{*}{36} & \multirow{4}{*}{$12-68$} \\
\hline 1 & 5 & 4 & & \\
\hline 2 & 4 & - & & \\
\hline 3 & 2 & - & & \\
\hline San Fco. de los Romo & 16 & 4 & \multirow{4}{*}{25} & \multirow{4}{*}{$8-52$} \\
\hline 1 & 5 & - & & \\
\hline 2 & 4 & 1 & & \\
\hline 3 & 7 & 3 & & \\
\hline San José de Gracia & 12 & 2 & \multirow{4}{*}{17} & \multirow{4}{*}{$3-49$} \\
\hline 1 & 4 & - & & \\
\hline 2 & 4 & 2 & & \\
\hline 3 & 4 & - & & \\
\hline Tepezalá & 12 & 5 & \multirow{4}{*}{42} & \multirow{4}{*}{$16-71$} \\
\hline 1 & 3 & 3 & & \\
\hline 2 & 5 & - & & \\
\hline 3 & 4 & 2 & & \\
\hline Total & 168 & 51 & 30 & $23-38$ \\
\hline
\end{tabular}

* IC: Intervalo de confianza 95\%. 
La frecuencia de animales positivos a Cryptosporidium spp., de acuerdo a las diferentes características registradas en la encuesta individual, se muestran en el Cuadro 2. La mayoría de los perros incluidos en el estudio fueron jóvenes, no mayores de 18 meses, y en estos mismos la frecuencia de la infección fue más importante; en los perros asociados a establos la frecuencia fue mayor que en los del área urbana (37 y $12 \%$, respectivamente). En ambas poblaciones, las hembras tuvieron más casos positivos que los machos, y en las muestras de excremento diarreico la frecuencia de casos positivos al parásito fue más alta que en las muestras de consistencia firme ( 31 y $11 \%$, respectivamente). En los perros asociados a los establos, la frecuencia de casos positivos al parásito de acuerdo al tipo de alimento que consumían, mostró valores de 22, 32 y $36 \%$ para alimento seco, preparado en casa y combinado, mientras que en los perros que consumía agua en bebederos exclusivos, la frecuencia fue más alta que en los que compartían el bebedero. Los perros vacunados contra alguna enfermedad infecciosa, generalmente rabia, y los no vacunados, tuvieron valores prácticamente iguales, mientras que los perros no desparasitados resultaron con mayor frecuencia de casos positivos al parásito.

Cuadro 2: Frecuencia de Cryptosporidium spp., diagnosticada por microscopía en perros del estado de Aguascalientes, México, de acuerdo a diferentes características

\begin{tabular}{|c|c|c|c|c|c|c|c|c|c|}
\hline \multirow[b]{2}{*}{$\overline{\text { Variable }}$} & \multicolumn{3}{|c|}{$\begin{array}{c}\text { Perros del área } \\
\text { urbana }\end{array}$} & \multicolumn{3}{|c|}{$\begin{array}{c}\text { Perros asociados } \\
\text { a establos }\end{array}$} & \multicolumn{3}{|c|}{ Total } \\
\hline & $n$ & Posit. & $\%$ & n & Posit & $\%$ & n & Posit. & $\%$ \\
\hline \multicolumn{10}{|l|}{ Edad (meses): } \\
\hline $0-6$ & 58 & 7 & 12 & 67 & 25 & 37 & 125 & 32 & 26 \\
\hline $7-18$ & 41 & 3 & 7 & 49 & 15 & 31 & 90 & 18 & 20 \\
\hline $19-66$ & 28 & 1 & 3 & 20 & 3 & 15 & 48 & 4 & 8 \\
\hline$>66$ & 17 & 2 & 12 & 32 & 8 & 25 & 49 & 10 & 20 \\
\hline \multicolumn{10}{|l|}{ Sexo: } \\
\hline$\overline{\text { Macho }}$ & 74 & 6 & 8 & 78 & 20 & 25.6 & 152 & 24 & 16 \\
\hline Hembra & 70 & 7 & 10 & 90 & 31 & 34 & 160 & 40 & 25 \\
\hline \multicolumn{10}{|l|}{ Excremento: } \\
\hline Firme & 90 & 5 & 5 & 77 & 14 & 18 & 167 & 19 & 11 \\
\hline Diarreico & 54 & 8 & 15 & 91 & 37 & 41 & 145 & 45 & 31 \\
\hline Alimento: & & na & & & & & & & \\
\hline Seco & & & & 37 & 8 & 22 & 37 & 8 & 22 \\
\hline Preparado & & & & 28 & 10 & 36 & 28 & 10 & 36 \\
\hline Combinado & & & & 103 & 33 & 32 & 103 & 33 & 32 \\
\hline Agua bebida: & & na & & & & & & & \\
\hline Exclusiva & & & & 98 & 42 & 43 & 98 & 42 & 43 \\
\hline Compartida & & & & 70 & 9 & 13 & 70 & 9 & 13 \\
\hline Medicina preventiva: & & na & & & & & & & \\
\hline Vacunación & & & & 121 & 36 & 30 & 121 & 36 & 30 \\
\hline No vacunación & & & & 47 & 15 & 32 & 47 & 15 & 32 \\
\hline Desparasitación & & & & 37 & 7 & 19 & 37 & 7 & 19 \\
\hline No desparasitación & & & & 131 & 44 & 33 & 131 & 44 & 33 \\
\hline
\end{tabular}

na: no aplica. 
El análisis de riesgos indicó que la variable excremento diarreico (OR, 3.2; IC95\% 1.06$9.79 P<0.03)$ en los perros de origen urbano y en los perros asociados a los establos (OR, 2.7; IC95\% 1.36-5.49 $P<0.001$ ), están relacionados con la frecuencia del parásito determinada por el análisis parasitoscópico. En ninguna de las otras variables analizadas fue posible identificar una asociación estadísticamente significativa.

\section{Discusión}

La literatura reporta que la prevalencia de la infección por Cryptosporidium en perros con dueño y callejeros en áreas urbanas y rurales se encuentra en un rango de 1 a $45 \%$, y que es más importante en cachorros que en animales adultos ${ }^{(9-11,15-21)}$. Aunque hay que considerar que cada estudio guarda características únicas en su diseño, prueba diagnóstica, región geográfica y condiciones ambientales. En el presente trabajo, la frecuencia de infección en perros del área urbana fue $9 \%$, lo que sitúa a la población estudiada en un nivel relativamente bajo, situación similar a la observada en perros con dueño domiciliados en la ciudad de México, en la cual se determinó una frecuencia de infección de $11.5 \%^{(22)}$. En los perros asociados a establos lecheros, la frecuencia determinada en el presente estudio fue de $30 \%$, sensiblemente más alta que la observada en los perros del área urbana; además, la presencia de animales positivos a la prueba se encuentra ampliamente distribuida en los sitios de muestreo, lo que confirma el carácter cosmopolita de Cryptosporidium; en la literatura se ha reportado una situación similar, en donde perros callejeros y con dueño de comunidades rurales tiene mayor frecuencia de infección que los perros de $\operatorname{ciudad}^{(9)}$. Hasta donde se conoce, no existen reportes en la literatura sobre la presencia de Cryptosporidium spp., en perros asociados a establos lecheros. Cabe mencionar que la criptosporidiosis en el ganado lechero de Aguascalientes se encuentra ampliamente distribuida, con una importante presencia en becerras de reemplazo, en las cuales se ha determinado una prevalencia entre 40 y $67 \%$, identificándose como única especie presente a C. parvum $^{(13,23)}$.

La frecuencia de Cryptosporidium en este estudio fue más importante en perros jóvenes, situación observada por otros autores $^{(15,22)}$; la información indica que la prevalencia a la infección tiende a disminuir en los cachorros infectados naturalmente conforme crecen $^{(24)}$; sin embargo, la edad no es considerada un factor de riesgo a contraer la infección ${ }^{(25,26)}$, como lo ha sido también en el presente trabajo.

El género de los animales no tuvo influencia en la presentación de casos positivos al protozoario en las poblaciones estudiadas, esto a pesar de que las hembras mostraron 
valores más altos que los machos; en otros estudios se ha observado este mismo resultado, $\mathrm{y}$ al igual que en este trabajo, no ha sido posible considerar al género como un factor de riesgo ${ }^{(25,27,28)}$.

La presencia de diarrea es considerada un signo clínico preponderante en la criptosporidiosis canina ${ }^{(8)}$, en el presente estudio las muestras de excremento consideradas por su consistencia como diarreicas, tuvieron mayor frecuencia de casos positivos que las heces firmes, y se identificó como un factor de riesgo, tanto en los perros de origen urbano (OR 3.2), como en los asociados a los establos (OR 2.7); cabe mencionar que las muestras provenían de animales clínicamente sanos, de tal forma que se considera que estos eran portadores asintomáticos. En la literatura se ha mencionado que los perros con diarrea excretan más ooquistes que aquellos que no muestran este signo clínico ${ }^{(29)}$, reconociéndose como un factor de riesgo ${ }^{(15,25,27)}$. Los perros asintomáticos o con episodios de diarrea esporádica son fuente de contaminación al suelo y agua en el entorno en que habitan, y debido a la resistencia de los ooquistes al medio ambiente, representan un riesgo de transmisión importante para el ser humano, así como para otros animales domésticos y silvestres, tanto en los ambientes rurales y de convivencia con el ganado, como en las ciudades y entornos peridomésticos ${ }^{(1)}$.

En los perros asociados a los establos lecheros, el tipo de alimentación no fue identificado como factor de riesgo, similar situación se observó en otro estudio ${ }^{(25)}$, pero debe notarse que los perros estudiados deambulan libremente en las instalaciones del establo lechero, de manera que pueden tener acceso a fetos y desechos placentarios así como desarrollar esporádicamente cacería de pájaros y pequeños roedores. El agua de bebida ha sido considerado un vehículo apropiado para la transmisión de Cryptosporidium, especialmente en las poblaciones humanas ${ }^{(1)}$; $\sin$ embargo, en este estudio solo se evaluó el uso del recipiente en que beben agua los perros, y esta variable no se identificó como factor de riesgo; en otro estudio, se evaluó la fuente del agua de bebida, sin embargo, no se encontró que fuera un factor de riesgo ${ }^{(25)}$.

La existencia de un programa mínimo de medicina preventiva en los perros asociados a los establos, reflejó que tanto los perros vacunados como los no vacunados sufren por igual la parasitosis; sin embargo, los que reciben un tratamiento antiparasitario presentaron menor frecuencia de infección que los que no lo reciben, aun así, esta variable no fue identificada como un factor de riesgo y tampoco de protección. De acuerdo a la información recabada en el presente estudio, es posible estimar que el ambiente prevalente en los establos lecheros favorece la transmisión y mantenimiento de la infección, además de que la densidad de perros en cada establecimiento, su movilidad y la interrelación que tienen con otros animales domésticos y silvestres también colaboren en esta situación; mientras que los perros en el ambiente urbano, la mayoría de ellos callejeros, se enfrentan a una situación ambiental diferente y resultan menos expuestos a la infección. 


\section{Conclusiones e implicaciones}

La infección por Cryptosporidium spp., está presente tanto en perros del ámbito urbano como en los que se encuentran asociados a establos lecheros, siendo que en los primeros la frecuencia es relativamente baja, mientras que en los segundos ésta debe de considerarse elevada. La distribución de la infección es amplia en las áreas rurales consideradas en el estudio y son reflejo de la capacidad infectiva del protozoario, siendo recomendable estudiar la relación perros-ganado bovino en la epidemiología de esta parasitosis. En tanto que debe de considerarse que ambas poblaciones de perros representan un riesgo para la transmisión al ser humano, ya que pueden ser portadores tanto de C. canis como de C. parvum.

\section{Agradecimientos}

Se agradece al personal del CCABA del municipio de Aguascalientes, así como a los ganaderos participantes en el estudio, su colaboración desinteresada en el desarrollo de este trabajo. El proyecto estuvo financiado por el Tecnológico Nacional de México.

\section{Literatura citada:}

1. Fayer R. General Biology. In: Fayer R, Xiao L. editors. Cryptosporidium and Cryptosporidiosis. $2^{\text {nd }}$ ed., Boca Raton, FL: CRC Press; 2008:1-35.

2. Robertson ID, Irwin PJ, Lymbery AJ, Thompson RCA. The role of companion animals in the emergence of parasitic zoonoses. Int J Parasitol 2000;(30):1369-1377.

3. Xiao L, Morgan UM, Limor J, Escalante A, Arrowood M, Shulaw W, et al. Genetic diversity within Cryptosporidium parvum and related Cryptosporidium species. Appl Environ Microbiol 1999;(65):3386-3391.

4. Fayer R, Trout JM, Xiao L, Morgan UM, Lal AA, Dubey JP. Cryptosporidium canis n. sp. from domestic dogs. J Parasitol 2001;(87):1415-1422. 
5. Hajdusek O, Ditrich O, Slapeta J. Molecular identification of Cryptosporidium spp. in animal and human hosts from the Czech Republic. Vet Parasitol 2004;(122):183192.

6. Lupo PJ. Cryptosporidium muris in a Texas canine population. Am J Trop Med Hyg 2008;(78):917-921.

7. Irwin PJ. Companion animal parasitology: a clinical perspective. Int J Parasitol 2002;(32):581-593.

8. Santin M. Clinical and subclinical infections with Cryptosporidium in animals. N Z Vet J 2013;(61):1-10.

9. Dubná S, Langrová I, Nápravník J, Jankovská I, Vadlejch J, Pekár S, et al. The prevalence of intestinal parasites in dogs from Prague, rural areas, and shelters of the Czech Republic. Vet Parasitol 2007;(145):120-128.

10. Jian F, Qi M, He X, Wang R, Zhang S, Dong H, et al. Occurrence and molecular characterization of Cryptosporodium in dogs in Henan Province, China. BMC Vet Res 2014;(10):26.

11. Ferreira JIGS, Pena HFJ, Azevedo SS, Labruna MB, Gennari SM. Occurrences of gastrointestinal parasites in fecal samples from domestic dogs in São Paulo, Brazil. Braz J Vet Parasitol 2016;(25):435-440.

12. INEGI. Instituto Nacional de Estadística, Geografía e Informática. Anuario Estadístico del Estado de Aguascalientes. México. 2011.

13. Castillo GC, Cruz-Vázquez C, López RR, Sánchez GM, Rosario CR, Vitela MI, et al. Frecuencia e identificación molecular de Cryptosporidium spp en becerras lactantes mantenidas en confinamiento en Aguascalientes, México. Téc Pecu Méx 2009;(47):425-434.

14. Hosmer DW, Lemeshow S, Sturdivant RX. Applied logistic regression. 3th ed. New Jersey, USA: Wiley; 2013.

15. Causapé AC, Quilez J, Sanchez-Acedo C, Del Cacho E. Prevalence of intestinal parasites, including Cryptosporidium parvum, in dogs in Zaragoza city, Spain. Vet Parasitol 1996;(67):161-167.

16. Abe N, Sawano Y, Yamada K, Kimata I, Iseki, M. Cryptosporidium infection in dogs in Osaka, Japan. Vet Parasitol 2002;(108):185-193.

17. Hackett T, Lappin MR. Prevalence of enteric pathogens in dogs of north-central Colorado. J Am Anim Hosp Assoc 2003;(39):52-56.

18. Ederli BB, Rodrigues MFG, Carvalho CB. Oocistos do gênero Cryptosporidium em cães domiciliados na Cidade de Campos dos Goytacazes, Estado do Rio de Janeiro. Rev Bras Parasitol Vet 2005;(14):129-131. 
19. Shukla R, Giraldo P, Kraliz A, Finnigan M, AL Sanchez. Cryptosporidium spp. and other zoonotic enteric parasites in a sample of domestic dogs and cats in the Niagara region of Ontario. Can Vet J 2006;(47):1179-1184.

20. Katagiri A, Oliveira-Sequeira TC. Prevalence of dog intestinal parasites and risk perception of zoonotic infection by dog owners in Sao Paulo State, Brazil. Zoonoses Public Health 2008;(55):406-413.

21. Overgaauw PA, Zutphen L, Hoek D, Yaya FO, Roelfsema J, Pinelli E, et al. Zoonotic parasites in fecal samples and fur from dogs and cats in The Netherlands. Vet Parasitol 2009;(163):115-122.

22. Martínez-Barbosa I, Gutiérrez M, Ruiz LA, Fernández AM, Gutiérrez EM, Aguilar JM, et al. Detección de Cryptosporidium spp y otros parásitos zoonoticos entéricos en perros domiciliados de la Ciudad de México. Arch Med Vet 2015;(47):347-353.

23. García-Romo D, Cruz-Vázquez C, Quezada-Tristán T, Silva-Peña E, ValdiviaFlores A, Vázquez-Flores S, et al. Prevalencia y factores de riesgo asociados a la infección por Cryptosporidium spp. en becerras lactantes en Aguascalientes, México. Vet Mex OA. 2014;(1):1 julio-septiembre.

24. Hamnes IS, Gjerde BK, Robertson LJ. A longitudinal study on the occurrence of Cryptosporidium and Giardia in dogs during their first year of life. Acta Vet Scand 2007;(49):22.

25. Ederli BB, Ederli NB, Oliveira FCR, Quirino CR, Carvalho C. Fatores de risco associados á infeccçãon por Cryptosporidium spp, em cäes domiciliados na cidade de Campos dos Goytacazes, estado do Rio de Janeiro, Brasil. Rev Bras Parasitol Vet 2008;(17):250-266.

26. Lallo MA, Bondan EF. Prevalência de Cryptosporidium sp. em cães de instituições da Cidade de São Paulo. Rev Saúde Púb 2006;(40):120-125.

27. Rodríguez BE, Manrique-Abril F, Pulido MM, Ospina-Díaz J. Frecuencia de Cryptosporidium spp en caninos de la ciudad de Tunja-Colombia. Rev MVZ Córdoba 2009;(14):1697-1704.

28. Mundim MJS, Rosa LAG, Hortêncio SM, Faria ESM, Rodrigues RM, Cury MC. Prevalence of Giardia duodenalis and Cryptosporodium spp. in dogs from different living conditions in Uberlândia, Brazil. Vet Parasitol 2007;(144):356-359.

29. Hill SL, Lappin MR. Cryptosporidiosis in the dog and cat. In: Bonagura J. editor. Kirk's Current Veterinary Therapy XII, Philadephia (PA):WB Saunders; 1995:728731. 